\title{
Breaks and outliers when modelling the volatility of the US stock market
}

Vasiliki Chatzikonstanti ${ }^{1}$

Department of Economics, University of Patras, University Campus, Rio 26504, Greece, e-mail: vchatz@upatras.gr

Aston Business School, Aston University, Aston Triangle, Birmingham B4 7ET, United Kingdom, e-mail: $\underline{\text { v.chatzikonstanti@aston.ac.uk }}$

${ }^{1}$ The author acknowledges funding from the University of Patras (Caratheodory Research Grant C.909). 


\title{
Breaks and outliers when modelling the volatility of the US stock market
}

\begin{abstract}
This study analyses volatility persistence of the US stock market, after taking into account the role of breaks and outliers. By employing a wavelet-based algorithm, it identifies several outliers which are comfortably associated with major events such as the 'Black Monday' and the Asian crisis. There is also evidence of clustering of breaks and a substantial variation in the properties of the identified segments.
\end{abstract}

Keywords: stock returns, outliers, structural breaks, GARCH

JEL codes: $C 12, C 22, C 59, G 10, G 15$ 


\section{Introduction}

Modelling the volatility of financial time series is a primary area of investigation in financial economics and econometrics. Understanding its behaviour is critical as it is a fundamental measure of risk and has considerable implications in numerous financial activities, such as asset pricing and portfolio selection. The most common properties of volatility, the fat (heavy)-tailed distribution and volatility clustering, have been thoroughly analysed particularly after the introduction of the seminal (G)ARCH models by Engle (1982) and Bollerslev (1986).

However, it is commonly accepted that financial markets have been severely influenced by extreme events such as financial crashes of foreign countries, wars, natural catastrophes and terrorist attacks. Consequently, it seems rather imperative that the modelling of financial time series (Charles and Darné, 2005; Bali and Guirguis, 2007) takes this into account. The two approaches that seem to be focal in the relevant strands of literature are to incorporate breaks in the mean and/or volatility dynamics and to identify and correct for the presence of outliers prior to fitting a particular model.

The presence of breaks and outliers may have undesirable effects on the estimates of the underlying volatility process. The impact of each one on volatility has been exhaustively examined. However, their joint impact on volatility has not been dealt with in depth yet. This article builds upon the notion that the behaviour of volatility is simultaneously affected by breaks in the mean and/or volatility dynamics and outliers. To this end, it proposes a methodological framework that integrates recent approaches for the detection of breaks and outliers. It employs a wavelet-based outlier detection method. The number and timing of potential 
breaks in outlier-corrected returns are identified non-parametrically. The actual detected breaks are confirmed using a variety of robustness tests that leads to segments with statistically different properties. Comparing the volatility persistence of the original returns, outlier-corrected returns, returns with sudden changes and segments determined by the break detection procedure will reveal to which extent breaks and outliers influence the behaviour of volatility.

In order to examine the research issue, this article utilizes returns from five US stock market indices over a long period. It finds outliers in all series associated with prominent events such as the "Black Monday", the Asian crisis or the recent financial crisis. The break analysis provides evidence of breaks in the mean and/or volatility dynamics in outlier-corrected returns, with noticeable differences across identified segments. The examination of GARCH models reveals that the series are highly persistent, if breaks are not accounted for, while ignoring outliers induces biases to GARCH parameters estimates.

The rest of the article is organised as follows. Section 2 briefly reviews the relevant literature. Section 3 contains the methodology. Section 4 provides an overview of the data. Section 5 presents the results and Section 6 concludes. 


\section{Literature Review}

The notion of breaks in the mean and/or volatility dynamics has attracted the attention of the research community at least since Diebold (1986) and Lamoureux and Lastrapes (1990). They demonstrate that the persistence in volatility is overestimated due to unaccounted for multiple structural changes. Mikosch and Starica (2004) point out that regime shifts in variance generate IGARCH effects. Hillebrand (2005) and Krämer and Azamo (2007) establish that neglecting structural breaks in the parameters of a GARCH model causes overestimation of persistence. More recently, Karoglou (2010) demonstrates that by accounting for breaks in the mean and/or volatility dynamics it is possible to arrive substantially closer to normality than by employing some GARCH-type models; and the two seem not to coexist.

Given the grave implications of the existence of breaks in modelling financial series, a number of procedures to detect them have been developed (see for example the LM-type tests of Andrews (1993), the Bai and Perron (1998 2003) methodology, as well as the more popular CUSUM tests of Inclàn and Tiao (1994)). Kokoszka and Leipus (1999, 2000), and subsequently Kim et al. (2000), Lee et al. (2004) and Sansó et al. (2004) modify the Inclan and Tiao CUSUM statistic to allow for dependent heterogeneous underlying processes, such as GARCH-type processes. The modification is based on scaling the CUSUM statistic with a long run variance estimator. This algorithm has been used for the detection of breaks in financial time series in a number of studies (Andreou and Ghysels, 2002; de Pooter and van Dijk, 2004; Rapach et al., 2008; McMillan and Wohar, 2011; Vivian and Wohar, 2012). In this article the identification of breaks in the mean and/or volatility dynamics is based on the modified version of the 
CUSUM statistic. It employs a non-parametric approach proposed by Kokoszka and Leipus (2000) scaled by a large number of different long run variance estimators to secure the findings against break under-reporting in contrast to other empirical studies that use a single long run variance estimator.

Outliers are observations that reflect extraordinary, infrequent events or rare large shocks that have important effects on modelling time series. In fact, the probability of these movements is much higher than what is expected by the normal distribution (and occur in clusters). The evidence of excess kurtosis observed in returns may reflect the existence of extreme stock market movements, as well.

The modelling of outliers seems to be the source of severe econometric issues, as their existence may erroneously suggest or hide true heteroscedasticity (van Dijk et al, 1999), lead to biases to the maximum likelihood estimators even in the case of a single outlier (Sakata and White, 1998; Carnero et al., 2007) and may also induce bias in the out-of-sample forecasts (Ledolter, 1989; Chen and Liu, 1993a; Franses and Ghijsels, 1999; Charles, 2008).

Furthermore, outliers in time series are related to "smearing" and the "masking" effects (see Bruce and Martin, 1989). The former is referred to the fact that the presence of some outliers may bias the diagnostics resulting in false identification of other outliers. The latter is associated to the occurrence of large outliers which prevent the identification of others. More recently, Rodrigues and Rubia (2011) show that outliers may induce large size distortions in break detection algorithms, as the existence of additive outliers may mask the presence and number of potential breaks. 
The severe problems of outliers' existence motivate the development of a number of methods to detect and accommodate them. For instance we refer the tests proposed by Tsay (1986, 1988), Chang et al. (1988) and Chen and Liu (1993b) based on ARMA models, while the recent literature focuses on detection and correction of outliers within the GARCH framework proposed by Franses and Ghijsels (1999), Charles and Darné (2005), Doornik and Ooms (2005), Zhang and King (2005), Bali and Guirguis (2007), Ané et al. (2008), Grané and Veiga(2010) and Hotta and Tsay (2012). Along these lines, this study focuses on the detection and correction of outliers to emphasize the existence of large shocks that may affect the stock markets. In doing so, it employs a wavelet procedure applied to the residuals of volatility models as proposed by Grane and Veiga (2010). This procedure is more reliable than others as detects less false outliers. Further, it avoids the joint estimation of the parameters of the underlying model and the detection of outliers, the presence of which may affect the parameters estimation.

\section{Methodology}

\section{Outlier detection}

This study applies a wavelet-based outlier detection algorithm proposed by Grané and Veiga (2010) to detect and correct outliers from the dataset. In the econometric literature, outliers are often distinguished to additive and innovative type. This study focuses on the detection of additive outliers. The innovative outliers are extreme disturbances that affect all observations after their occurrence (see Pena, 2001) and seem to overlap with the notion of breaks. For that reason they are treated as synonymous. 
The procedure of Grane and Veiga (2010) is chosen since it is based on the residuals of an estimated model, which is estimated only once, in contrast to other proposed algorithms who suggest an iterative procedure (see for instance Franses and Ghijsels, 1999 and Doornik and Ooms, 2005). The adopted algorithm allows for a recursively manner of outliers' identification. It avoids the joint estimation of the parameters of the underlying model and the outliers, the presence of which may affect the parameters estimation ${ }^{2}$. Moreover, the wavelet-based outlier procedure appears to be more reliable than others such as Franses and Ghijsels (1999) and Doornik and Ooms (2005) procedures, since it reduces the probability of false detected outliers. Finally, the classification of an observation as an outlier is based on critical values obtained by simulation and are not derived from rules of thumb or asymptotics (Bilen and Huzurbazar, 2002).

The detection of outliers is based on the detail coefficients from the discrete wavelet transformation of the residuals of a volatility model, such as a GARCH model which is common for financial time series. The errors can follow a standard normal or a Student's t distribution ${ }^{3}$ (see Grane and Veiga, 2010 for an extensive analysis of the procedure).

An observation is classified as an outlier if the absolute value of the detailed coefficients is greater than a threshold value. The threshold is defined as the $95^{\text {th }}$ percentile of the distribution of the maximum of the detailed coefficients (in absolute value) obtained by simulated similar processes of the same size. The simulation-based derivation of critical values allows assuming different distribution in case the standard assumption of normally distributed residuals is

\footnotetext{
2 The procedure allows for only one detected outlier at a time, which means that when an outlier is detected then the series is corrected and the adjusted series is used for the detection of a new outlier. 3 The algorithm can be extended to other error distributions, such as the Generalized Error Distribution.
} 
questionable. Therefore, the coefficients whose absolute value exceeds the threshold value results in a set of identified outlier positions, $S=\left\{s_{1}, \ldots, s_{n}\right\}$. The final step of the procedure involves the correction of the identified outliers and reconstruction of the series, which are performed by applying the inverse discrete wavelet transform.

\section{Detecting breaks in volatility}

Regarding the number and timing of the potential breaks, this article employs a CUSUM-type test designed to detect breaks in unconditional volatility. Specifically, it utilizes a modified version of the Inclan and Tiao (1994) algorithm, as proposed by Kokoszka and Leipus (2000) that allows for dependent processes. The Inclan and Tiao (1994) procedure is designed for i.i.d. processes, which is a very strong assumption for financial time series. As shown by and Andreou and Ghysels (2002), Sanso et al. (2004) and de Pooter and van Dijk (2004), the Inclan and Tiao (1994) test can be substantially oversized when the series follow a dependent process, such as a GARCH model. Therefore, it is employed a nonparametric adjustment proposed by Kokoszka and Leipus (2000) that includes various dependent underlying processes (henceforth KL).

Let denote the process of interest $X_{t}=r_{t}^{2}$, with $r_{t}$ the returns of the series, the KL statistic, $K L_{T}$, is given by

$$
K L_{T}=\max _{1 \leq k \leq T}\left|\frac{1}{\hat{\sigma}_{L R}} D_{T}(k)\right|
$$

where $D_{T}(k)=\frac{1}{\sqrt{T}} \sum_{t=1}^{k} X_{t}+\frac{k}{T \sqrt{T}} \sum_{t=1}^{T} X_{t}$ and $\hat{\sigma}_{L R}$ a consistent estimator of the long run variance estimator. Under certain mild regularity conditions the asymptotic distribution of the KL statistic is given by $\sup _{0 \leq s \leq 1}|B(s)|$, where $B(s)$ is 
a standard Brownian bridge, while the $90 \%, 95 \%$ and $99 \%$ asymptotic critical values are $1.22,1.36$ and 1.63 respectively.

The KL statistic was fundamentally designed to test for a single break; however it can be applied in a sequential manner to identify multiple breaks, similar to the ICSS algorithm proposed by Inclan and Tiao (1994). This study adopts the sequential segmentation procedure to detect multiple breaks based on the KL statistic. First, the entire sample is tested for the presence of a single break. If a break is detected, the sample is split into two sub-samples with break date set as the split point. Second, each sub-sample is examined separately for a single break. If a new change point is detected, the sub-sample is further divided into two new segments. This procedure continues until no more breaks are found in any of the sub-samples. The significance level in each testing step must take into account the number of breaks that have already been detected. This is achieved by using a significance level of $\alpha /(N+1)$, where $a=0.05$ is the nominal significance level at the first step and $N$ the number of breaks, when testing for the $(N+1)$ break. Third, the identified set of breaks is ordered and cross-checked using adjacent breakpoints. If a previously identified break point does not reject the null, it is dropped from the final set of breaks. Further restrictions can be imposed on the algorithm, for example we can allow for a maximum number of breaks and a minimum distance between adjacent breaks (de Pooter and van Dijk, 2004). ${ }^{4}$

The KL statistic requires a consistent estimator $\hat{\sigma}_{L R}^{2}$ of the long run variance of $\left\{X_{t}\right\}$. There are a number of procedures in order to estimate the long run variance, such as estimators which depend on the kernel function one uses. For

\footnotetext{
4 Following de Pooter and van Dijk (2004) we impose a minimum distance between adjacent breaks of 3 business months to prevent breaks from being identified unrealistically close.
} 
that purpose, this study employs seven different estimators for the long run variance $\sigma_{L R}^{2}$ :

(i) the Newey and West (1987) estimator.

(ii) the Newey and West (1994) estimator.

(iii) the Andrews (1991) quadratic spectral estimator.

(iv) the Vector Autoregression Heteroscedasticity and Autocorrelation Consistent (VARHAC) estimator of Den Haan and Levin (1997) using the AIC information criterion.

(v) the Vector Autoregression Heteroscedasticity and Autocorrelation Consistent (VARHAC) estimator of Den Haan and Levin (1997) using the BIC information criterion.

(vi) the Andrews (1991) Bartlett kernel estimator using an ARMA(1,1) model.

(vii) the Andrews (1991) Bartlett kernel estimator using an AR(1) model.

As the number of detected breaks will differ across long run variance estimators, the actual detected breaks will be confirmed using a variety of methods designed to test for the equality of means and/or variances of two contiguous segments. Specifically, the tests of the "Awarding breakdates" stage as proposed by Karoglou (2010) are adopted. In this stage, the standard t-test and the Satterthwaite-Welch t-test are used for the equality of means and the standard Ftest, the Siegel-Tukey test with continuity correction (Siegel and Tukey (1960) and Sheskin (1997)), the adjusted Bartlett test (see Sokal and Rohlf (1995) and Judge et al. (1985)), the Levene test (1960) and the Brown-Forsythe test (1974) for the equality of variances. 


\section{Volatility model}

A standard $A R(1)-G A R C H(1,1)$ model $^{5}$ for the returns is employed as it is flexible enough to assess the most important stylised facts of the data and to examine the volatility persistence of financial time series. The conditional mean is given by

$r_{t}=\mu+\rho r_{t-1}+\varepsilon_{t}$

where

$\varepsilon_{t}=\eta_{t} \sigma_{t}$

$\varepsilon_{t} \sim N\left(0, \sigma_{t}^{2}\right)$

$\eta_{t} \sim$ i.i.d. $N(0,1)$

and the conditional variance of $\varepsilon_{t}$ is given by

$\sigma_{t}^{2}=\omega+\alpha \varepsilon_{t-1}^{2}+\beta \sigma_{t-1}^{2}$

The parameters satisfy at least $\omega>0$ and $\alpha, \beta \geq 0$ to ensure that the conditional variance is positive and the existence of the GARCH process. The process is stationary if $\alpha+\beta<1$. The sum of $\alpha$ and $\beta$ indicates the persistence of a shock. The typical value of the sum, especially for stock market return series, is very close to one, implying that shocks are highly persistent. If $\alpha+\beta=1$, then one has an integrated GARCH (IGARCH) process where shocks have a permanent effect on volatility. Beyond analysing the persistence of a process through the sum of the $\alpha$ and $\beta$ parameters, it is used a volatility measure with intuitive interpretation to quantify the volatility persistence, the half-life of a shock. This measure is calculated as $\ell=\ln (0.5) / \ln (\alpha+\beta)$ and measures the period of time

\footnotetext{
5 The autoregressive parameter is included in the conditional mean, if series show evidence of autocorrelation indicated by the autocorrelation and partial autocorrelation functions.
} 
(days in our study) over which a shock to volatility decays to half its original size. For a stationary $\operatorname{GARCH}(1,1)$ the unconditional variance of $\varepsilon_{t}$ is given by $\omega /(1-\alpha-\beta)$. Notice that when $\alpha=0, \beta$ is unidentified and set equal to zero then the series is characterized by conditional homoscedasticity. The parameters are estimated using the quasi maximum likelihood estimation (QMLE).

The standard GARCH model is augmented with dummy variables to account for the identified breaks in unconditional volatility. In particular, we estimate

$r_{t}=\mu+\rho r_{t-1}+\varepsilon_{t}$

where

$\varepsilon_{t}=\eta_{t} \sigma_{t}$

$\varepsilon_{t} \sim N\left(0, \sigma_{t}^{2}\right)$

$\eta_{t} \sim$ i.i.d.N $(0,1)$

and the conditional variance of $\varepsilon_{t}$ is given by

$\sigma_{t}^{2}=\omega_{1}+d_{1} D_{1}+\cdots+d_{n} D_{n}+\varepsilon_{t-1}^{2}+\beta \sigma_{t-1}^{2}$

where $D_{i}$ are the dummy variables taking the value of one from each point of structural break in variance and zero elsewhere.

\section{Data}

The data set consists of daily closing values of five US stock market indices, namely the S\&P 500, MSCI world US (MSWRLD), NASDAQ Composite, Dow Jones Industrial Average (DJIA) and NYSE Composite. The data are obtained from Datastream. The sample period runs from 3 January 1983 to 19 March 2013 resulting in a total of 7782 observations. Table 1 provides some descriptive statistics for the stock index (log) returns. 


\section{[Table 1 around here]}

The stock market returns demonstrate the usual properties of financial data; specifically, a small mean is dominated by a large standard deviation. The NASDAQ index is the most volatile series, while the MSWRLD the least. Moreover, returns are highly non-normal, showing evidence of negative skewness and significant kurtosis. The kurtosis ranges from 11.612 for the NASDAQ index to 45.769 for the DJIA, supporting the existence of outliers. The Lagrange Multiplier (LM) test indicates that all series exhibit significant evidence of ARCH effect. The outlier-adjusted returns still exhibit excess skewness, kurtosis and conditional heteroscedasticity. However, the excess skewness and kurtosis are dramatically reduced. For instance, in the S\&P 500 and DJIA indices, their values decreased more than $50 \%$.

Table 1 also presents the pair-wise correlations among the stock market indices. These estimated pair-wise correlation coefficients are all greater than 0.7 and statistically significant at $1 \%$ confirming that the indices are positively correlated. The NYSE and S\&P 500 indices are tightly linked with a correlation of 0.98. The DJIA and S\&P 500 and NYSE and DJIA pairs exhibit high correlation as well, 0.964 and 0.952 respectively. The lowest correlation, 0.732 , is reported between NASDAQ and MSWRLD indices.

\section{Empirical Results}

Table 2 presents the timing of the identified outliers by employing the wavelet-based procedure ${ }^{6}$ to the residuals series from a $\operatorname{GARCH}(1,1)$ model with

\footnotetext{
${ }^{6}$ A threshold value of $k_{1}^{0.05}=4.3521$ computed from 20,000 Monte Carlo replications of size $n=$ 7700 is used.
} 
Gaussian errors ${ }^{7}$ for each stock market index along with their percentage change and the events that may be associated with. A total of 18 outliers is detected which is relatively low compared to other studies (for instance see Charles and Darné, 2012), ranging from two for MSWRLD and NASDAQ indices to six for the DJIA index. It is worth mentioning that the S\&P 500 and NYSE indices exhibit exactly the same outliers; this pair has the highest correlation. On the other hand the pair with the lowest correlation, NASDAQ and MSWRLD, has no common outliers. The detected outliers are negative, with the exception of the outlier in the MSWRLD index in January 17, 1991 which is in accordance with the literature that negative outliers occur more frequently than positive ones (Jansen and de Vries, 1991). Noteworthy the majority of these negative outliers are common among the examined indices. This can be explained by the fact that markets appear to be higher related during periods of extreme negative variations (see for instance Longin and Solnik, 2001; Ang and Bekaert, 2002; Ané and Kharoubi, 2003).

\section{[Table 2 around here]}

Beyond the mere analysis of the detection of outliers, this study examines the time of these outlying observations. The timing of the detected outliers reveals that stock market indices exhibit outliers in common dates. The first outlier corresponds to the day known as "Black Monday", identified in four indices namely the S\&P 500, MSWRLD, DJIA and NYSE. This corresponds to the highest loss during the examined time period for the stock market indices $-20.41 \%$, $9.84 \%,-22.61 \%$ and $-19.17 \%$ respectively ${ }^{8}$. The second outlier identified in the S\&P 500, DJIA and NYSE indices on October 13, 1989 corresponds to the mini

\footnotetext{
${ }^{7}$ In the case of a volatility model with the errors following a t-student distribution no outliers are detected, in accordance with the findings of Grane and Veiga (2010).

${ }^{8}$ On October 19, 1987 the fall of the DJIA is recorded as the largest one-day percentage decline from 1928 to 2010 (Charles and Darne, 2012).
} 
stock market crash from the failure of the buyout deal for United Continental Holding (UAL) Corporation, the parent company of United Airlines. The average decline this day was $6.28 \%$. The outlier in the MSWRLD index on January 17, 1991 caused a rise of $4.88 \%$ in the index and is associated with the Gulf War I, specifically the Operation Desert Storm. The outlier on November 15, 1991 in DJIA index coincides with bad economic statistics, related to the stagnation of US economic activity. A prominent outlier is identified in the S\&P 500, NASDAQ, DJIA and NYSE indices on October 27, 1997 due to Asian crisis, resulting in an average reduction of $6.91 \%$ in the indices. The terrorist attacks in the US on September 11th, 2001 seem to have affected the stock markets. On September 17, 2001, the day that the US stock market reopened, an outlier is detected in DJIA index, resulting in a decline of 7.13\%. Finally, the outlier of February 27, 2007 in the S\&P 500, NASDAQ, DJIA and NYSE indices corresponds to the day of the decline of the Shanghai Stock Exchange and the news regarding the recession in the US economy, with an average decrease of $3.56 \%$.

\section{[Figure 1 around here]}

Utilizing the outlier-corrected series, the study proceeds with the analysis aiming at the identification of breaks. The outlier-corrected instead of the original returns are adapted, since Rodrigues and Rubia (2011) demonstrate that additive outliers may mask the presence and the number of potential breaks detected by the CUSUM-type statistics. Figure 1 presents the returns for S\&P 500 and DJIA along with \pm 3 -standard-deviation bands for each of the regimes defined by the structural breaks. We have included both the identification of breaks in case of no outliers corrected returns and the outlier corrected returns in order to demonstrate how the existence of outliers may mask the identification of breaks. If outliers are not 
accounted for, two breaks in the unconditional volatility of the S\&P 500 index are identified. The number of breaks increases to eight for the outlier adjusted returns. For the DJIA no breaks are detected for the non-outlier adjusted returns and two when the outliers are taken into account. Similar results hold for the rest of the indices ${ }^{9}$. Table 3 reports the results on the timing of the identified breaks and Table 4 presents descriptive statistics of each identified segment ${ }^{10}$.

\section{[Table 3 around here]}

The break detection reveals that the changes in unconditional volatility of the US stock market returns can be captured at least by two breaks, as in the case of DJIA while the dynamics of the NASDAQ index seems to be more complex, as nine breaks have been identified. It is interesting to note that the number of identified breaks in volatility is different despite the high correlation between the indices. However the timing of common identified breaks coincides. It is worth mentioning that most of the breakdates are attributed to major events, providing evidence about the economic impact of these events. For instance, four indices, namely S\&P 500, MSWRLD, NASDAQ and NYSE, show a break in July 2007 and DJIA in October 2007 which is associated with the beginning of the Financial Crisis of 2007-2008. Four of the examined series, S\&P 500, MSWRLD, NASDAQ and NYSE, exhibit another break in September 2008, the month that the financial institutions crisis hits its peak. Several major American institutions such as Lehman Brothers, Merrill Lynch, Fannie Mae, Freddie Mac, Washington Mutual, Wachovia, Citigroup and AIG, either failed or were subject to government

\footnotetext{
${ }^{9}$ Results for the remaining indices are available upon request.

${ }^{10}$ Due to space limitations we do not report the results from the tests for the equality of mean and variance of contiguous segments; however the relevant results are available upon request.
} 
takeover. Finally, all indices have a break in March-June 2009, which is related to European debt crisis.

\section{[Table 4 around here]}

Another interesting aspect of the break analysis is the existence of a large segment from 1983 to $1997 / 1998$ in the S\&P 500, MSWRLD, NASDAQ and NYSE indices which spans on average fifteen and a half years (about the $50 \%$ of the total number of observations). A second large segment (1997-2007) is also identified in MSWRLD index that accounts for the $33 \%$ of the total sample. Moreover, it is worth noting that a larger segment that runs from 1983 to 2007 that covers twenty-five years (more than the $80 \%$ of the total observations) is identified in the DJIA index. These large segments appear to be relatively calm, in contrast to the small segment during 2007/08-2009 (spanning on average 266 days) identified in all examined indices which reveals an episode of significant high volatility with standard deviation over $2 \%$.

Regarding the descriptives for the identified segments as represented in Table 4, substantial differences across segments are noticed. In ten segments the distribution is positively skewed and negatively in the other segments, while almost all segments are leptokurtic. Furthermore, the normality assumption is not rejected in nine segments. Lastly, the $26 \%$ of the segments does not exhibit conditional heteroscedasticity. These results indicate the importance of breaks in the mean and/or volatility dynamics when fitting a model.

The final part of the analysis is devoted to the volatility persistence of stock markets returns. A benchmark GARCH model is employed to the original returns, the outlier-corrected returns, the returns with sudden changes in variance and to the identified segments. Table 5 summarises the relevant results. Inspection of the 
parameter estimates over the full sample (original returns) reveals that the GARCH processes are highly persistent with the sum of the $\alpha$ and $\beta$ parameters ranging from 0.985 (MSWRLD) to 0.994 (NASDAQ), in line with the extant literature, and the median value equals to 0.998 . Using half-life for the examination of the persistence the half-lives range from 47 to 124 days and the median (mean) value across series is $59(71)$.

\section{[Table 5 around here]}

The degree of persistence is slightly increased when the outlier corrected returns are employed, as measured by the sum of the $\alpha$ and $\beta$ parameters, ranging from 0.985 to 0.995 . The increase is more noticeable when examining the half-life measure. The number of days that a shock decreases to its half size is increased on average by $30 \%$. The median value of the sum of the parameters is 0.99 and corresponds to a half-life of 80 days. Furthermore, the unconditional variance is reduced by $9 \%$ on average for the outlier adjusted returns. However such a reduction can be considered rather remarkable as there are only 2 to 6 outliers in 7782 observations. The DJIA index that has the highest number of identified outliers, exhibits the highest decrease in the unconditional variance. An interesting finding present in all series is that the value of $\alpha$ parameter decreases and the value of the $\beta$ parameter increases in the outlier-corrected returns. Notably the reduction of $\alpha$ parameter is much higher than the increase of the $\beta$ parameter. This imply that large innovations have larger short run effects and financial time series revert to their long run trends relatively quickly. Therefore, the uncounted outliers imply that the $\alpha$ parameter is overestimated and the $\beta$ parameter is underestimated ${ }^{11}$.

11 This finding is in accordance with the existing literature, see for instance Charles and Darne (2006), Carnero et al. (2007, 2012) and Franses and Ghijsels (1999). 
Once the identified breaks are incorporated into the benchmark GARCH model by including the dummy variables in the variance equation the volatility persistence, as measured by the sum of the $\alpha$ and $\beta$ parameters, is considerably reduced in all cases ${ }^{12}$. Among the examined indices, NASDAQ shows the largest decline in volatility persistence from 0.995 (outlier adjusted returns) to 0.95 . The smallest reduction is observed for the DJIA, from 0.991 to 0.989 . Alternatively, by quantifying the decrease in volatility persistence through the half-life values $\ell$, the half-life values range from 14 (NASDAQ) to 63 (DJIA) days. It is worth mentioning that the median half-life percentage reduction among series is equal to $60 \% .^{13}$

Examination of parameter estimates across sub-samples reveals that the decrease in persistence is often sizable. In the extreme, there are sub-samples where the persistence vanishes, the estimates of $\alpha$ is equal to zero and the subsamples are characterized by conditional homoscedasticity ${ }^{14}$. The persistence in the identified segments as measured by the sum of the $\alpha$ and $\beta$ parameters is reduced with a median (mean) value across segments equal to $0.973(0.967)$. The reduction of the persistence is substantial if the half-life measure is employed; notably only in three segments the half-life is greater than the full sample half-life. Across the segments the half-life ranges from 5 to 72 days and the median (mean) value is 26 (31). Therefore, the results regarding the volatility persistence in identified segments reveal a substantial reduction from the GARCH $(1,1)$ process

\footnotetext{
12 The findings are similar to other studies, see for example, Aggarwal et al. (1999) Ewing and Malik (2010), and Wang and Moore (2009).

13 Overall, our results are in accordance with Lamoureux and Lastrapes (1990) who argue that standard GARCH model overestimates the persistence in volatility since relevant sudden changes in variance are ignored.

${ }^{14}$ Similar results for sub-sample GARCH estimates have been employed in Rapach et al. (2008), McMillan and Wohar (2011) and Vivian and Wohar (2012) among others.
} 
that does not allow for breaks in the mean and/or volatility dynamics. The reduction in the volatility persistence is remarkably even when compared with the results from GARCH model augmented with dummy variables.

If we focus on volatility persistence at segments during the recent financial crisis, as defined by the break on September 2008, we notice that the segment that covers the period from July 2007 to September 2008 are characterized by homoscedasticity for all indices. On the other hand, on the segment that covers the period from Sept 2008 to May/June 2009, the persistence seems to be quite different across the indices. The highest persistence $(0.981)$ is noticed at the NASDAQ index and the lowest (0.964 and 0.965) at MSWRLD and NYSE indices.

\section{Conclusion}

This study examines the volatility modelling, emphasising on the volatility persistence, by evaluating the impact of breaks in the mean and/or volatility dynamics and outliers. Employing a wavelet-based outlier detection method, it finds several outliers in the US stock market indices (S\&P 500, MSWRLD, NASDAQ, DJIA and NYSE). The identified outliers are associated with high impact events such as the financial crises, wars and terrorist attacks. Due to the fact that outliers may bias the presence and the timing of breaks, a CUSUM-type statistic to detect breaks in the outlier corrected data is applied. The dynamics of the examined series are quite complex with the number of identified breaks ranging from two to nine. The break analysis reveals clustering of breaks in periods with high uncertainty such as the recent financial crisis and the European debt crisis. The properties of the identified segments appear to vary substantially, 
pointing the importance of accounting for outliers and breaks. Regarding the volatility persistence, the existence of outliers bias the parameters estimates of the volatility persistence, while ignoring the possible breaks lead in spuriously high estimates of volatility persistence. Therefore, the volatility modelling is noticeably improved when breaks and outlier are taken into account.

A further interesting direction would be to study the volatility of other stock markets through the analysis of breaks and outliers and examine the possible transmission mechanisms across markets. 


\section{References}

Aggarwal, R., Inclan, C. and Leal, R. (1999) Volatility in Emerging Stock Markets, Journal of Financial and Quantitative Analysis, 34, 33-55.

Andreou, E. and Ghysels, E. (2002) Detecting multiple breaks in financial market volatility dynamics, Journal of Applied Econometrics, 17, 579-600.

Andrews, D.W.K. (1991) Heteroscedasticity and autocorrelation consistent covariance matrix estimation, Econometrica, 59, 817-58.

Andrews, D.W.K. (1993) Tests for parameter instability and structural change with unknown change point, Econometrica, 61, 821-56.

Ang, A. and Bekaert, G. (2002) International asset allocation with regime shifts, Review of Financial Studies, 11, 1137-87.

Ané, T. and Kharoubi, C. (2003) Dependence Structure and Risk Measure, The Journal of Business, 76, 411-38.

Ané, T., Ureche-Rangau, L., Gambet, J.B. and Bouverot, J. (2008) Robust Outlier Detection for Asia-Pacific Stock Index Returns, Journal of International Financial Markets, Institutions and Money, 18, 326-43.

Bai, J. and Perron, P. (1998) Estimating and testing linear models with multiple structural changes, Econometrica, 66, 47-78.

Bai, J. and Perron, P. (2003) Computation and analysis of multiple structural change models., Journal of Applied Econometrics, 18, 1-22.

Bali, R. and Guirguis, H. (2007) Extreme observations and non-normality in $\mathrm{ARCH}$ and GARCH, International Review of Economics and Finance, 16, $332-46$. 
Bollerslev, T. (1987) A conditionally heteroskedastic time series model for speculative prices and rates of return, Review of Economics and Statistics, 69, 542-47.

Brown, M.B. and Forsythe, A.B. (1974) Robust tests for equality of variances, Journal of the American Statistical Association, 69, 364-67.

Bruce, A.G. and Martin, R.D. (1989) Leave-k-Out Diagnostics for Time Series, Journal of the Royal Statistical Society. Series B (Methodological), 51, 363424.

Carnero, M.A., Pena, D. and Ruiz, E. (2007) Effects of outliers on the identification and estimation of GARCH models, Journal of Time Series Analysis, 28, 471-97.

Carnero, M.A., Pena, D. and Ruiz, E. (2012) Estimating GARCH volatility in the presence of outlier, Economics Letters, 114, 86-90.

Charles, A. (2008) Forecasting volatility with outliers in GARCH models, Journal of Forecasting, 27, 551-65

Charles, A. and Darné, O. (2005) Outliers and GARCH models in daily financial data, Economics Letters, 86, 347-52.

Charles, A. and Darné, O. (2006) Large shocks and the September $11^{\text {th }}$ terrorist attacks on international stock markets, Economic Modelling, 23, 683-98.

Charles, A. and Darné, O. (2012) Large Shocks in the Volatility of the Dow Jones Industrial Average Index: 1928-2010, Working Papers hal-00678932, HAL.

Chang, I., Tiao, G.C. and Chen, C. (1988) Estimation of time series parameters in the presence of outliers, Technometrics, 30, 193-204. 
Chen, C. and Liu, L. (1993a) Forecasting time series with outliers, Journal of Forecasting, 12, 13-35.

Chen, C. and Liu, L. (1993b) Joint estimation of model parameters and outlier effects, Journal of American Statistical Association, 88, 284-97.

de Pooter, M. and van Dijk, D. (2004) Testing for changes in volatility in heteroscedastic time series - a further examination, Erasmus University Rotterdam, Econometric Institute Report 2004-38/A.

Den Haan, W.J. and Levin, A. (1997) A practitioner's guide to robust covariance matrix estimation in Handbook of Statistics: Robust Inference, (Eds) G.S. Maddala and C.R. Rao, Elsevier, 291-341.

Diebold, F. (1986) Modeling the persistence of conditional variances: A comment, Econometric Reviews, 5, 51-6.

Doornik, J.A. and Ooms, M. (2005) Outlier detection in GARCH models, Working paper, Nuffield College, Oxford.

Engle, R.F. (1982) Autoregressive conditional heteroskedasticity, with estimates of the variance of the United Kingdom inflation, Econometrica, 50, 9871007.

Ewing, B. T. and Malik, F. (2010), Estimating Volatility Persistence in Oil Prices Under Structural Breaks, Financial Review, 45, 1011-1023

Franses, P.H. and Ghijsels, H. (1999) Additive outliers, GARCH and forecasting volatility, International Journal of Forecasting, 15, 1-9.

Grané, A. and Veiga, H. (2010) Wavelet-based detection of outliers in financial time series, Computational Statistics and Data Analysis, 54, 2580-93.

Hillebrand, E. (2005) Neglecting parameter changes in GARCH models, Journal of Econometrics, 129, 121-38. 
Hotta, L.K. and Tsay, R.S. (2012) Outliers in GARCH processes. In Economic time series: modelling and seasonality. W.R. Bell, S.H. Holan, and T.S. McElroy (Eds.), Boca Raton: Chapman \& Hall, 337-58.

Inclan, C. and Tiao, C.G. (1994) Use of cumulative sums of squares for retrospective detection of changes of variance, Journal of the American Statistical Association, 89, 913-23.

Jansen, D. W. and De Vries, C.G. (1991) On the frequency of large stock returns: Putting booms and busts into perspective, The Review of Economics and Statistics, 71, 18-24.

Judge, G.G., Griffiths, W.E., Hill, R.C., Ltkepohl, H. and Lee, T.-C. (1985) The theory and practice of Econometrics. NewYork: JohnWiley and Sons.

Karoglou, M, (2010) Breaking down the non-normality of daily stock returns, European Journal of Finance, 16, 79-95.

Kim, S., Cho, S. and Lee, S. (2000) On the CUSUM test for parameter changes in GARCH(1,1) models, Communication in Statistics, Theory and Methods, 29, 445-62.

Kokoszka, P. and Leipus, R. (1999) Testing for parameter changes in ARCH models, Lithuanian Mathematical Journal, 39, 231-47.

Kokoszka, P. and Leipus, R. (2000) Change-point estimation in ARCH models, Bernoulli, 6, 1-28.

Krämer, W. and Azamo, B.T. (2007) Structural change and estimated persistence in the GARCH(1,1)-model, Economics Letters, 97, 17-23. 
Lamoureux, C. and Lastrapes, W. (1990) Persistence in variance structural change and the GARCH model, Journal of Business and Economic Statistics, 8, 225-34.

Ledolter, J. (1989) The effect of additive outliers on the forecasts from ARIMA models, International Journal of Forecasting, 23, 1-40.

Lee, S., Tokutsu, Y. and Maekawa, K. (2004) The CUSUM test for parameter change in regression models with ARCH errors, Journal of the Japan Statistical Society, 34, 173-88.

Levene, H. (1960) Contributions to Probability and Statistics: Essays in Honor of Harold Hotelling, in Ingram Olkin, Sudhish G. Ghurye, Wassily Hoeffding, William G. Madow, Henry B. Mann. California: Stanford University Press. Longin, F. and Solnik, B. (2001) Extreme correlations in international equity markets, Journal of Finance, 56, 649-76.

McMillan, D. and Wohar, M.E. (2011) Structural breaks in volatility: The case of UK sector returns, Applied Financial Economics, 21, 1079-93.

Mikosch, T. and Starica, C. (2004) Non-stationarities in financial time series, the long-range dependence and the IGARCH effects, Review of Economics and Statistics, 86, 378-90.

Newey, W. and West, K. (1987) A simple positive semi-definite heteroscedasticity and autocorrelation consistent covariance matrix, Econometrica, 55, 703-08.

Newey, W. and West, K. (1994) Automatic lag selection in covariance matrix estimation, Review of Economic Studies, 61, 631-53. 
Pena, D. (2001) Outliers, Influential Observations and Missing data. In: A course in time series, Pena, D., G.C. Tiao and R.S. Tsay (eds). John Wiley, New York.

Rapach, D., J. Strauss and Wohar, M.E. (2008) Forecasting stock return volatility in the presence of structural breaks in Forecasting in the Presence of Structural Breaks and Model Uncertainty, (Eds) D.E. Rapach and M.E. Wohar, Emerald Group Publishing Limited, pp. 381-416.

Rodrigues, P.M.M. and Rubia, A. (2011) The effects of additive outliers and measurement errors when testing for structural breaks in variance, Oxford Bulletin of Economics and Statistics, 73, 449-68.

Sakata, S. and White, H. (1998) High breakdown point conditional dispersion estimation with application to S\&P500 daily returns volatility, Econometrica, 66, 529-67.

Sansó, A., Aragó, V. and Carrion, J.L. (2004) Testing for Changes in the Unconditional Variance of Financial Time Series, Revista de Economía financier, 4, 32-53.

Sheskin, D.J. (1997) Handbook of Parametric and Nonparametric Statistical Procedures, Boca Raton, FL: CRC Press.

Siegel, S. and Tukey, J.W. (1960) A Nonparametric Sum of Ranks Procedures for Relative Spread in Unpaired Samples, Journal of the American Statistical Association, 55, 429-44.

Sokal, R.R. and Rohlf, F.J. (1995) Biometry: the principles and practice of statistics in biological research. New York, NY:W.H. Freemand and Co.

Tsay, R.S. (1986) Time series model specification in the presence of outliers, Journal of the American Statistical Association, 81, 131-41. 
Tsay, R.S. (1988) Outliers, level shifts, and variance changes in time series, Journal of Forecasting, 7, 1-20.

Zhang, X. and King, M. (2005) Influence Diagnostics in Generalized Autoregressive Conditional Heteroscedasticity Processes, Journal of Business and Economic Statistics, 23, 118-29.

van Dijk, D., Franses, P.H. and Lucas, A. (1999) Testing for ARCH in the presence of additive outliers, Journal of Applied Econometrics, 14, 539-62.

Vivian, A. and Wohar, M.E. (2012) Commodity volatility breaks, Journal of International Financial Markets, Institutions and Money, 22, 395-422.

Wang, P. and Moore, T. (2009) Sudden changes in volatility: The case of five central European stock markets, Journal of International Financial Markets, Institutions and Money, 19, 33-46. 


\section{Tables}

Table 1. Descriptive statistics and correlation matrix

\begin{tabular}{|c|c|c|c|c|c|}
\hline Returns & $S \& P 500$ & $M S W R L D$ & $N A S D A Q$ & DJIA & NYSE \\
\hline Mean & 0.031 & 0.028 & 0.033 & 0.034 & 0.030 \\
\hline St. Dev. & 1.141 & 0.918 & 1.380 & 1.117 & 1.099 \\
\hline Skewness & -1.271 & -0.544 & -0.237 & -1.683 & -1.306 \\
\hline Kurtosis & 31.655 & 14.418 & 11.612 & 45.769 & 31.350 \\
\hline $\mathrm{JB}$ & \multicolumn{2}{|c|}{$271759.80 * 43197.63 *$} & \multicolumn{3}{|c|}{$24430.51 * 604390.70 * 266165.00 *$} \\
\hline $\mathrm{LM}(10)$ & $74.8 *$ & $259.2 *$ & $192.8^{*}$ & $47.4^{*}$ & $105.9 *$ \\
\hline \multicolumn{6}{|c|}{ Outlier Corrected Returns } \\
\hline Mean & 0.033 & 0.028 & 0.034 & 0.037 & 0.033 \\
\hline St. Dev. & 1.117 & 0.912 & 1.377 & 1.083 & 1.078 \\
\hline Skewness & -0.467 & -0.427 & -0.219 & -0.395 & -0.586 \\
\hline Kurtosis & 15.005 & 12.871 & 11.603 & 15.839 & 17.023 \\
\hline $\mathrm{JB}$ & $47609.59 *$ & $32233.43 *$ & $24367.87 *$ & $54338.23^{*}$ & $65026.21 *$ \\
\hline $\mathrm{LM}(10)$ & $199.6^{*}$ & $251.8^{*}$ & $195.6^{*}$ & $178.7 *$ & $257 *$ \\
\hline \multicolumn{6}{|c|}{ Correlation matrix } \\
\hline$S \& P 500$ & 1 & & & & \\
\hline$M S W R L D$ & 0.810 & 1 & & & \\
\hline$N A S D A Q$ & 0.844 & 0.732 & 1 & & \\
\hline DJIA & 0.964 & 0.776 & 0.756 & 1 & \\
\hline NYSE & 0.980 & 0.837 & 0.794 & 0.952 & 1 \\
\hline
\end{tabular}

Note: $*$ Denotes significance at $1 \%$.

Table 2. Identified outliers

\begin{tabular}{|lll|}
\hline Date & Stock Market Index (Percentage change) & Event \\
\hline $19 / 10 / 1987$ & $\begin{array}{l}\text { S\&P 500 (-20.41), MSWRLD (-9.48), } \\
\text { DJIA (-22.61), NYSE (-19.17) }\end{array}$ & Black Monday \\
$13 / 10 / 1989$ & S\&P 500 (-6.13), DJIA (-6.91), NYSE (-5.80) & Rejection of repurchase plan of United Airlines \\
$17 / 01 / 1991$ & MSWRLD (4.88) & Operation Desert Storm \\
$15 / 11 / 1991$ & DJIA (-3.93) & Bad economic statistics; Fear of economic stagnation \\
$27 / 10 / 1997$ & $\begin{array}{l}\text { S\&P 50 (-6.87), NASDAQ (-7.02), } \\
\text { DJIA (-7.18), NYSE (-6.57) }\end{array}$ & Asian Cris is \\
$17 / 09 / 2001$ & DJIA (-7.13) & The September 11t terrorist attack \\
$27 / 02 / 2007$ & $\begin{array}{l}\text { S\&P 500 (-3.47), NASDAQ (-3.86), } \\
\text { DJIA (-3.29), NYSE (-3.63) }\end{array}$ & Fall of Shanghai Stock Exchange; fear of reces sion \\
\hline
\end{tabular}


Table 3. Identified breaks in volatility

\begin{tabular}{|c|c|c|c|c|c|c|c|}
\hline Newey-West (1987) & Newey-West (1994) & Quadratic Spectral & VARHAC AIC & VARHAC BIC & Bartlett ARMA & Bartlett AR & Adopted \\
\hline \multicolumn{8}{|l|}{$S \& P 500$} \\
\hline - & 20/07/1998 & 20/07/1998 & - & - & 20/07/1998 & 26/03/1997 & 26/03/1997 \\
\hline- & $28 / 04 / 2003$ & $28 / 04 / 2003$ & - & - & - & $03 / 05 / 2002$ & $20 / 07 / 1998$ \\
\hline- & 09/07/2007 & 09/07/2007 & - & - & - & $25 / 07 / 2003$ & $03 / 05 / 2002$ \\
\hline- & $12 / 09 / 2008$ & $12 / 09 / 2008$ & - & - & - & 09/07/2007 & $28 / 04 / 2003$ \\
\hline- & 01/06/2009 & 01/06/2009 & - & - & - & $12 / 09 / 2008$ & $25 / 07 / 2003$ \\
\hline- & - & - & - & - & - & $01 / 06 / 2009$ & 09/07/2007 \\
\hline - & - & - & - & - & - & - & $12 / 09 / 2008$ \\
\hline- & - & - & - & - & - & - & $01 / 06 / 2009$ \\
\hline \multicolumn{8}{|l|}{$M S W R L D$} \\
\hline 23/07/2007 & $16 / 10 / 1997$ & 16/10/1997 & - & - & 23/07/2007 & $16 / 10 / 1997$ & 16/10/1997 \\
\hline - & 03/09/2008 & 03/09/2008 & - & - & - & 03/09/2008 & $23 / 07 / 2007$ \\
\hline - & $13 / 05 / 2009$ & $13 / 05 / 2009$ & - & - & - & $04 / 05 / 2009$ & $03 / 09 / 2008$ \\
\hline - & - & - & - & - & - & $01 / 08 / 2011$ & $04 / 05 / 2009$ \\
\hline - & - & - & - & - & - & $20 / 12 / 2011$ & $01 / 08 / 2011$ \\
\hline - & - & - & - & - & - & - & $20 / 12 / 2011$ \\
\hline \multicolumn{8}{|l|}{$N A S D A Q$} \\
\hline 27/07/1998 & 27/07/1998 & 27/07/1998 & 27/07/1998 & $15 / 10 / 1997$ & 27/07/1998 & $15 / 10 / 1997$ & $15 / 10 / 1997$ \\
\hline $16 / 12 / 2002$ & $16 / 12 / 2002$ & $16 / 12 / 2002$ & $16 / 12 / 2002$ & 03/01/2000 & $16 / 12 / 2002$ & $03 / 01 / 2000$ & 27/07/1998 \\
\hline - & $23 / 07 / 2007$ & $23 / 07 / 2007$ & - & $16 / 12 / 2002$ & - & $23 / 04 / 2001$ & $03 / 01 / 2000$ \\
\hline - & $12 / 09 / 2008$ & $12 / 09 / 2008$ & - & - & - & $16 / 12 / 2002$ & $23 / 04 / 2001$ \\
\hline - & 01/06/2009 & 01/06/2009 & - & - & - & $18 / 08 / 2004$ & $16 / 12 / 2002$ \\
\hline - & - & - & - & - & - & $23 / 07 / 2007$ & $18 / 08 / 2004$ \\
\hline - & - & - & - & - & - & $12 / 09 / 2008$ & $23 / 07 / 2007$ \\
\hline - & - & - & - & - & - & $01 / 06 / 2009$ & $12 / 09 / 2008$ \\
\hline- & - & - & - & - & - & - & $01 / 06 / 2009$ \\
\hline \multicolumn{8}{|l|}{ DJIA } \\
\hline - & $31 / 10 / 2007$ & $31 / 10 / 2007$ & - & - & - & $31 / 10 / 2007$ & $31 / 10 / 2007$ \\
\hline- & $21 / 04 / 2009$ & $21 / 04 / 2009$ & - & - & - & $21 / 04 / 2009$ & $21 / 04 / 2009$ \\
\hline \multicolumn{8}{|l|}{ NYSE } \\
\hline 23/07/2007 & 23/07/2007 & 23/07/2007 & 23/07/2007 & 23/07/2007 & 23/07/2007 & 20/07/1998 & $20 / 07 / 1998$ \\
\hline - & $18 / 05 / 2009$ & $18 / 05 / 2009$ & - & - & - & 03/09/2008 & $23 / 07 / 2007$ \\
\hline - & - & - & - & - & - & 03/06/2009 & $03 / 09 / 2008$ \\
\hline - & - & - & - & - & - & - & $18 / 05 / 2009$ \\
\hline
\end{tabular}

Note: The breakdates are significant at $5 \%$. 
Table 4. Descriptive statistics for the identified segments

\begin{tabular}{|c|c|c|c|c|c|c|c|c|}
\hline Segments & Subperiod & Obs. & Mean & St. Dev. & Skewness & Kurtosis & $\mathrm{JB}$ & $\mathrm{LM}(10)$ \\
\hline$S \& P 500$ seg. 1 & 03/01/1983-26/03/1997 & 3712 & 0.0505 & 0.8792 & -1.3626 & 28.9235 & $105088.50 *$ & $91.10 *$ \\
\hline$S \& P 500 \mathrm{seg} .2$ & 27/03/1997-20/07/1998 & 343 & 0.1354 & 0.9938 & 0.2222 & 4.7179 & $45.00 *$ & 0.54 \\
\hline$S \& P 500 \mathrm{seg} .3$ & $21 / 07 / 1998-03 / 05 / 2002$ & 989 & -0.0099 & 1.3050 & -0.1087 & 5.0025 & $167.19 *$ & $4.49 *$ \\
\hline$S \& P 500 \mathrm{seg} .4$ & 06/05/2002-28/04/2003 & 256 & -0.0624 & 1.7133 & 0.3831 & 3.2860 & $7.13 * *$ & $4.47^{*}$ \\
\hline$S \& P 500 \mathrm{seg} .5$ & $29 / 04 / 2003-25 / 07 / 2003$ & 64 & 0.1370 & 0.9819 & -0.1010 & 2.7127 & 0.33 & $2.11 * *$ \\
\hline$S \& P 500 \mathrm{seg} .6$ & 28/07/2003-09/07/2007 & 1031 & 0.0435 & 0.6551 & -0.0830 & 3.4499 & $9.88 *$ & $3.67 *$ \\
\hline$S \& P 500$ seg. 7 & $10 / 07 / 2007-12 / 09 / 2008$ & 309 & -0.0654 & 1.2990 & -0.0505 & 3.3538 & 1.74 & 0.71 \\
\hline$S \& P 500 \mathrm{seg} .8$ & $15 / 09 / 2008-01 / 06 / 2009$ & 186 & -0.1523 & 3.2244 & -0.0045 & 4.1533 & $10.31 *$ & $3.56^{*}$ \\
\hline$S \& P 500$ seg. 9 & 02/06/2009-19/03/2013 & 991 & 0.0501 & 1.1131 & -0.4494 & 6.7477 & $613.31 *$ & $24.33 *$ \\
\hline MSWRLD seg. 1 & 03/01/1983-16/10/1997 & 3858 & 0.0479 & 0.6970 & -0.5818 & 19.1562 & $42176.94 *$ & $204.77^{*}$ \\
\hline MSWRLD seg. 2 & $17 / 10 / 1997-23 / 07 / 2007$ & 2547 & 0.0209 & 0.8819 & -0.1497 & 5.3157 & $578.6 *$ & $38.64 *$ \\
\hline MSWRLD seg. 3 & $24 / 07 / 2007-03 / 09 / 2008$ & 292 & -0.0760 & 1.0060 & -0.0066 & 3.3266 & 1.3 & 1.27 \\
\hline MSWRLD seg. 4 & 04/09/2008-04/05/2009 & 173 & -0.2069 & 2.7154 & -0.0783 & 3.8823 & $5.79 * * *$ & $4.85^{*}$ \\
\hline MSWRLD seg. 5 & 05/05/2009-01/08/2011 & 585 & 0.0585 & 0.9953 & -0.1812 & 4.4478 & $54.29 *$ & $2.75^{*}$ \\
\hline MSWRLD seg. 6 & 02/08/2011-20/12/2011 & 101 & -0.1074 & 1.8919 & -0.2558 & 2.9493 & 1.11 & 0.76 \\
\hline MSWRLD seg. 7 & $21 / 12 / 2011-19 / 03 / 2013$ & 325 & 0.0627 & 0.7565 & 0.0924 & 4.0472 & $15.31 *$ & 1.13 \\
\hline NASDAQ seg. 1 & 03/01/1983-15/10/1997 & 3857 & 0.0521 & 0.8470 & -1.6896 & 25.2817 & $81622.98 *$ & $171.08 *$ \\
\hline NASDAQ seg. 2 & 16/10/1997-27/07/1998 & 203 & 0.0852 & 1.1473 & -0.1717 & 3.5508 & 3.56 & 0.98 \\
\hline NASDAQ seg. 3 & 28/07/1998-03/01/2000 & 375 & 0.2025 & 1.8590 & -0.4879 & 4.4538 & $47.90 *$ & $3.55^{*}$ \\
\hline NASDAQ seg. 4 & 04/01/2000-23/04/2001 & 340 & -0.2048 & 3.1757 & 0.3099 & 3.9724 & $18.84 *$ & $2.24 * *$ \\
\hline NASDAQ seg. 5 & $24 / 04 / 2001-16 / 12 / 2002$ & 430 & -0.0897 & 2.1310 & 0.2386 & 3.0458 & 4.12 & 1.26 \\
\hline NASDAQ seg. 6 & $17 / 12 / 2002-18 / 08 / 2004$ & 437 & 0.0614 & 1.2888 & 0.0699 & 3.2908 & 1.9 & $2.58 *$ \\
\hline NASDAQ seg. 7 & $19 / 08 / 2004-23 / 07 / 2007$ & 763 & 0.0532 & 0.8171 & -0.0822 & 3.4575 & $7.51 * *$ & $3.69 *$ \\
\hline NASDAQ seg. 8 & $24 / 07 / 2007-12 / 09 / 2008$ & 299 & -0.0581 & 1.4237 & 0.0453 & 2.9616 & 0.12 & 0.56 \\
\hline NASDAQ seg. 9 & 15/09/2008-01/06/2009 & 186 & -0.1142 & 3.2088 & 0.0206 & 3.8543 & $5.67 * * *$ & $2.36 * *$ \\
\hline NASDAQ seg. 10 & 02/06/2009-19/03/2013 & 991 & 0.0574 & 1.2173 & -0.3893 & 6.1551 & $436.07 *$ & $20.61 *$ \\
\hline DJIA seg. 1 & $03 / 01 / 1983-31 / 10 / 2007$ & 6477 & 0.0448 & 0.9853 & -0.5999 & 17.0992 & $54035.89 *$ & $120.53 *$ \\
\hline DJIA seg. 2 & 01/11/2007-21/04/2009 & 384 & -0.1454 & 2.2120 & 0.2324 & 6.6385 & $215.27 *$ & $11.28 *$ \\
\hline DJIA seg. 3 & $22 / 04 / 2009-19 / 03 / 2013$ & 1020 & 0.0584 & 1.0198 & -0.3501 & 6.1718 & $448.40 *$ & $23.39 *$ \\
\hline NYSE seg. 1 & 03/01/1983-20/07/1998 & 4055 & 0.0541 & 0.8148 & -1.3236 & 28.9105 & $114614.60 *$ & $104.42 *$ \\
\hline NYSE seg. 2 & 21/07/1998-23/07/2007 & 2350 & 0.0208 & 0.9911 & -0.0994 & 6.1644 & $984.37 *$ & $34.15^{*}$ \\
\hline NYSE seg. 3 & 24/07/2007-03/09/2008 & 292 & -0.0692 & 1.2880 & -0.0877 & 3.2228 & 0.98 & 0.87 \\
\hline NYSE seg. 4 & 04/09/2008-18/05/2009 & 183 & -0.1876 & 3.4483 & -0.0517 & 3.8237 & $5.25 * * *$ & $3.92 *$ \\
\hline NYSE seg. 5 & $19 / 05 / 2009-19 / 03 / 2013$ & 1001 & 0.0430 & 1.2204 & -0.4224 & 6.3230 & $490.32 *$ & $22.92 *$ \\
\hline
\end{tabular}

Note: * Denotes significance at $1 \%$.

** Denotes significance at $5 \%$.

$* * *$ Denotes significance at $10 \%$. 
Table 5. QMLE for AR(1)-GARCH(1,1) models

\begin{tabular}{|c|c|c|c|c|c|c|c|}
\hline & $\hat{\rho}$ & $\widehat{\omega}$ & $\hat{\alpha}$ & $\widehat{b}$ & $\hat{\alpha}+\widehat{b}$ & $\ell$ & $\widehat{\omega} /(1-\hat{\alpha}-\widehat{b})$ \\
\hline \multicolumn{8}{|l|}{$S \& P 500$} \\
\hline Original Returns & - & $0.013(0.005)$ & $0.078(0.019)$ & $0.912(0.020)$ & 0.990 & 68.878 & 1.331 \\
\hline Outlier Corrected Returns & - & $0.009(0.002)$ & $0.067(0.010)$ & $0.926(0.010)$ & 0.993 & 96.882 & 1.219 \\
\hline Returns with dummies & & $0.017(0.004)$ & $0.067(0.010)$ & $0.907(0.013)$ & 0.974 & 26.203 & 0.663 \\
\hline Segment 1 & $0.054(0.017)$ & $0.007(0.004)$ & $0.050(0.018)$ & $0.941(0.021)$ & 0.990 & 72.501 & 0.741 \\
\hline Segment 2 & - & $0.985(0.103)$ & 0.000 & 0.000 & - & - & 0.985 \\
\hline Segment 3 & - & $0.087(0.038)$ & $0.066(0.022)$ & $0.883(0.032)$ & 0.949 & 13.199 & 1.694 \\
\hline Segment 4 & - & $0.183(0.120)$ & $0.086(0.033)$ & $0.848(0.063)$ & 0.934 & 10.125 & 2.772 \\
\hline Segment 5 & - & $0.968(0.156)$ & 0.000 & 0.000 & - & - & 0.968 \\
\hline Segment 6 & - & $0.022(0.010)$ & $0.044(0.013)$ & $0.904(0.026)$ & 0.949 & 13.123 & 0.426 \\
\hline Segment 7 & $-0.185(0.052)$ & $1.619(0.137)$ & 0.000 & 0.000 & - & - & 1.619 \\
\hline Segment 8 & $-0.177(0.067)$ & $0.150(0.132)$ & $0.084(0.033)$ & $0.896(0.032)$ & 0.979 & 32.911 & 7.210 \\
\hline Segment 9 & - & $0.031(0.010)$ & $0.104(0.024)$ & $0.870(0.023)$ & 0.974 & 26.242 & 1.197 \\
\hline \multicolumn{8}{|l|}{ MSWRLD } \\
\hline Original Returns & $0.172(0.012)$ & $0.012(0.004)$ & $0.104(0.023)$ & $0.881(0.024)$ & 0.985 & 47.073 & 0.853 \\
\hline Outlier Corrected Returns & $0.174(0.012)$ & $0.011(0.003)$ & $0.094(0.014)$ & $0.892(0.016)$ & 0.985 & 47.161 & 0.760 \\
\hline Returns with dummies & $0.176(0.012)$ & $0.018(0.004)$ & $0.102(0.016)$ & $0.859(0.022)$ & 0.961 & 17.566 & 0.455 \\
\hline Segment 1 & $0.211(0.017)$ & $0.037(0.014)$ & $0.147(0.037)$ & $0.766(0.062)$ & 0.914 & 7.673 & 0.430 \\
\hline Segment 2 & $0.162(0.020)$ & $0.008(0.003)$ & $0.067(0.012)$ & $0.923(0.013)$ & 0.990 & 67.615 & 0.736 \\
\hline Segment 3 & - & $1.009(0.090)$ & 0.000 & 0.000 & - & - & 1.009 \\
\hline Segment 4 & - & $0.234(0.135)$ & $0.091(0.033)$ & $0.874(0.031)$ & 0.964 & 19.118 & 6.585 \\
\hline Segment 5 & $0.125(0.042)$ & $0.027(0.016)$ & $0.054(0.024)$ & $0.918(0.030)$ & 0.972 & 24.535 & 0.965 \\
\hline Segment 6 & - & $3.544(0.492)$ & 0.000 & 0.000 & - & - & 3.544 \\
\hline Segment 7 & $0.102(0.049)$ & $0.565(0.055)$ & 0.000 & 0.000 & - & - & 0.565 \\
\hline \multicolumn{8}{|l|}{ NASDAQ } \\
\hline Original Returns & $0.112(0.012)$ & $0.013(0.003)$ & $0.098(0.015)$ & $0.897(0.014)$ & 0.994 & 123.762 & 2.257 \\
\hline Outlier Corrected Returns & $0.112(0.012)$ & $0.012(0.003)$ & $0.096(0.014)$ & $0.899(0.014)$ & 0.995 & 127.305 & 2.181 \\
\hline Returns with dummies & $0.116(0.012)$ & $0.029(0.006)$ & $0.098(0.016)$ & $0.851(0.022)$ & 0.950 & 13.460 & 0.572 \\
\hline Segment 1 & $0.258(0.017)$ & $0.031(0.011)$ & $0.124(0.031)$ & $0.828(0.041)$ & 0.952 & 14.086 & 0.638 \\
\hline Segment 2 & - & $1.310(0.147)$ & 0.000 & 0.000 & - & - & 1.310 \\
\hline Segment 3 & - & $0.496(0.182)$ & $0.164(0.075)$ & $0.694(0.070)$ & 0.858 & 4.522 & 3.494 \\
\hline Segment 4 & - & $10.097(0.930)$ & 0.000 & 0.000 & - & - & 10.097 \\
\hline Segment 5 & - & $4.531(0.313)$ & 0.000 & 0.000 & - & - & 4.531 \\
\hline Segment 6 & - & $1.828(0.309)$ & 0.000 & 0.000 & - & - & 1.828 \\
\hline Segment 7 & - & $0.027(0.013)$ & $0.029(0.012)$ & $0.929(0.023)$ & 0.959 & 16.468 & 0.660 \\
\hline Segment 8 & $-0.130(0.053)$ & $1.974(0.157)$ & 0.000 & 0.000 & - & - & 1.974 \\
\hline Segment 9 & $-0.167(0.066)$ & $0.135(0.145)$ & $0.073(0.034)$ & $0.908(0.036)$ & 0.981 & 35.591 & 6.983 \\
\hline Segment 10 & - & $0.038(0.013)$ & $0.092(0.023)$ & $0.881(0.024)$ & 0.973 & 25.729 & 1.415 \\
\hline \multicolumn{8}{|l|}{ DJIA } \\
\hline Original Returns & - & $0.015(0.005)$ & $0.080(0.020)$ & $0.908(0.019)$ & 0.988 & 59.412 & 1.323 \\
\hline Outlier Corrected Returns & - & $0.010(0.002)$ & $0.069(0.009)$ & $0.923(0.010)$ & 0.991 & 78.330 & 1.139 \\
\hline Returns with dummies & & $0.011(0.003)$ & $0.068(0.010)$ & $0.921(0.010)$ & 0.989 & 63.110 & 1.000 \\
\hline Segment 1 & - & $0.008(0.002)$ & $0.057(0.011)$ & $0.936(0.012)$ & 0.993 & 94.100 & 1.029 \\
\hline Segment 2 & $-0.151(0.046)$ & $0.054(0.033)$ & $0.105(0.021)$ & $0.885(0.018)$ & 0.990 & 72.501 & 5.670 \\
\hline Segment 3 & - & $0.026(0.008)$ & $0.112(0.024)$ & $0.864(0.023)$ & 0.976 & 28.219 & 1.053 \\
\hline \multicolumn{8}{|l|}{ NYSE } \\
\hline Original Returns & $0.036(0.012)$ & $0.014(0.005)$ & $0.084(0.019)$ & $0.904(0.020)$ & 0.988 & 57.905 & 1.151 \\
\hline Outlier Corrected Returns & $0.041(0.012)$ & $0.009(0.002)$ & $0.073(0.010)$ & $0.919(0.010)$ & 0.992 & 82.565 & 1.067 \\
\hline Returns with dummies & $0.042(0.012)$ & $0.011(0.003)$ & $0.073(0.010)$ & $0.910(0.012)$ & 0.983 & 40.650 & 0.680 \\
\hline Segment 1 & $0.097(0.017)$ & $0.007(0.004)$ & $0.054(0.019)$ & $0.935(0.023)$ & 0.989 & 62.971 & 0.643 \\
\hline Segment 2 & - & $0.011(0.004)$ & $0.072(0.012)$ & $0.917(0.013)$ & 0.989 & 61.591 & 0.997 \\
\hline Segment 3 & $-0.174(0.057)$ & $1.589(0.135)$ & 0.000 & 0.000 & - & - & 1.589 \\
\hline Segment 4 & $-0.153(0.067)$ & $0.366(0.212)$ & $0.087(0.031)$ & $0.878(0.030)$ & 0.965 & 19.63 & 10.547 \\
\hline Segment 5 & - & $0.031(0.011)$ & $0.096(0.024)$ & $0.883(0.023)$ & 0.979 & 32.315 & 1.457 \\
\hline
\end{tabular}

Note: Standard errors are given in parentheses. 


\section{Figures}
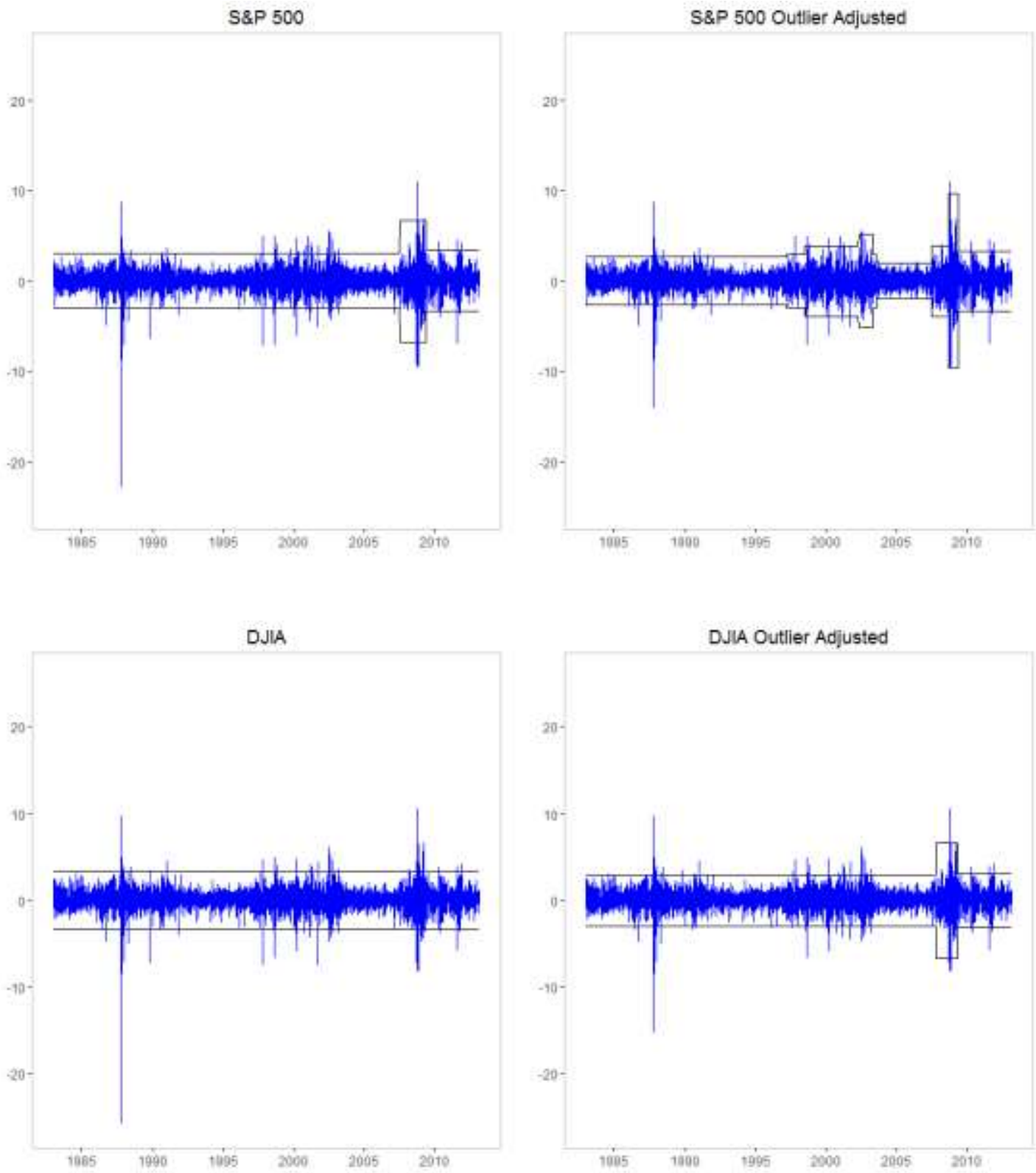

Figure 1. Returns and outlier adjusted returns for S\&P 500 and DJIA indices along with \pm 3 -standard-deviation bands for the regimes as defined by the structural breaks. 